\title{
Herbal Medicines In The Treatment Of Post-Traumatic Stress Disorder: A Review
}

\author{
L. Vasileva ${ }^{1,2}$, D. Getova \\ ${ }^{1}$ Department of Pharmacology and clinical pharmacology, Faculty of Medicine, Medical University Plovdiv \\ ${ }^{2}$ Department of Pharmacology and drug toxicology, Faculty of Pharmacy, Medical University Plovdiv
}

\section{INTRODUCTION}

There is an opinion that until 2020 depression will be the second highest in morbidity after cardiovascular diseases representing significant socioeconomic burden. The pathophysiology of major depressive disorder (MDD) is complicated and it seems that there are various overlapping biological factors [1]. In recent decades a fundamental prerequisite of the pathophysiology of MDD highlights monoamine impairment (dysfunction in the monoamine receptors expressionand activity), decreased monoamine production or secondary intermediary(eg.G proteins or cyclic AMP) [2]. Further attention is also given to the role of neuroendocrine abnormalities including excess cortisol and its hindering effects on neurogenesis by reducing the resultant of the brain derived neurotropic factor (BDNF) as well as impaired endogenous opioid function, changes in GABA-ergic and / or glutamatergic transmission, in the production of cytokines or steroids, and abnormal circadian rhythm [2-4]

Post-Traumatic Stress Disorder (PTSD) resulting from experience of a traumatic event that causes fear, helplessness and horror. Characterized by three groups of symptoms: 1)hyperamnesia to parent traumatic event often repeated experience in the form of flashbacks and nightmares, repulsive memories that can be provoked by sensorimotor stimuli, such as noise reminiscent of the patient to the traumatic event, and impaired memory to the peri-traumatic events; 2) hyper excitability characterized by excessive startle, hyper vigilance, and irritability; and 3) avoidance behavior of everything associated with the trauma. Symptoms must persist for at least four weeks before being diagnosed.PTSD affects a subset of people exposed to traumatic events at some point in their lives [5].

In the present review we aim to discuss the current known symptoms, targets and mechanisms of PTSD and future perspectives for phytotherapy.

\section{Neuronal networks and substrates involved in PTSD}

As a concept, PTSD can be considered as maladaptation to a traumatic stressor expressed in changed conditions and learning attenuation behavioral sensitization and changes in brain regions and neurotransmitter systems closely associated with these processes. A large amount of literature now focuses on central role of the corticolimbic circuit in PTSD [6]. Neuroimages have reported abnormalities in the prefrontal cortex, the hippocampus and the amygdala in patients with PTSD. These neural circuits are involved in alleged anomalies in conditional training and sensitization in PTSD. For example, insufficient control of the prefrontal cortex to the amygdala are believed to have an important role in injured attenuation of fear-associated memories and executive control in fear-driven responses. Weaker hippocampal-prefrontal communication can also enhance memory deficits in PTSD, manifested in poor control of fear contingent answers. Many of these pathways are involved in different phases of suspected development of PTSD or initial development of fear conditioning learning or attenuation.

Consideration of these pathways involves the participation of certain neurotransmitters systems: projection from the prefrontal cortex to the amygdala or dopamine or acetylcholine incoming channels in the amygdala, which glutamatergic nature. Thus unsatisfactory control of the prefrontal cortex to the amygdala suggests involvement of glutamatergic system in PTSD, directly or indirectly. For example, it is believed that the attenuation of fear requires prefrontal activation of intercalation cells in the amygdala, GABA interneurons, which inhibit local activation and express a unique receptor profile. Therefore, the level of amygdala other subcores can influence through glutamatergic and GABA interactions exported GABA system as a potential target for therapy for PTSD.

More recently, other functional way involved in responses to acute stress has emerged that includes indirect way to inhibit the hypothalamic-pituitary-adrenal (HPA) axis. Prefrontal cortex inhibits the HPA axis by glutamatergic projection to theBed Nucleus of Stria Terminalis(BNST), part of the extended amygdala, which activate GABA inhibitory projection of BNST to neurons producing corticotrophin releasing hormone (CRF) in hippotalamus.This time, there may be special treatment because patients with PTSD showed increased activity 
of some other systems than the HPA axis suggesting the possible usefulness of certain substances that reduce theCRFsystemandhormonesHPAaxisinthetreatmentofPTSD.

\section{Neural networks and substrates as targets for reducing symptoms of PTSD}

New experimental data obtained in animal models of PTSD, with the use of three different stress factors that repeating aspects of PTSD found, i.e. enhanced negative feedback HPA axis and enhanced startle reactivity, also showed that glutamatergic system may change over time. Contrary to the increased release of glutamate after exposure to acute stress, it was found that prolonged stress leads to weakened prefrontal levels of glutamate in rats. This reduction in glutamate levels may resemble an alleged reduced prefrontal activity in patientswithPTSD.

Hyper responsiveness of noradrenergic system seems to be retained in patients with PTSD and suggest that mediates the common excitement symptoms and sleep disturbances reported in this disorder. Increased noradrenergic mediation in the amygdala and hippocampus may facilitate recall of aversive memories.

Another monoaminergic system associated with PTSD is serotonergic. Polymorphism of the 5-HT transporter, which is in connection with the traumatic events in adults and injuries in childhood is likely to be a factor for susceptibility to PTSD. Moreover, the stress increased levels of 5-HT in several forebrain areas, including frontal cortex, hippocampus and amygdala. SSRIs are also useful in the treatment of the disorder at least in some patients, suggesting that serotonin plays a role in the pathogenesis of this disorder. Therefore, neuromodulators'systems that can be a target of pharmacological approaches for the treatment of PTSDincludeserotonergic,noradrenergic,GABA-ergicandglutamatergic.

Finally, there may be different pharmacological approaches, depending on whether the treatment is focused on those aspects in the development of PTSD (preventive measures during the time immediately after the traumatic event) or the objective is the treatment of chronic post-traumatic stress disorder (reduction of symptoms). If the aim is to prevent the consolidation of the memories associated with the trauma at an early stage, by blockade of glutamatergic activity or facilitate attenuation of these memories once they are already reinforced by increased glutamatergic functionality, i.e. opposing mechanisms of action may be used, depending on the phase of the disease to be treated.

\section{PHARMACOLOGICAL TREATMENT OPTIONS FOR PTSD}

The pharmacotherapy for PTSD should aimat : 1) reducing core PTSD symptoms in all three clusters(re-experience, avoidance and numbing and increased arousal);2) reducingfunctional impairment and disability; 3) improvinglife quality; 4) improving resilience to stress or trauma; 5) reducing co-morbidity such as depression, other anxietydisorders and substance abuse; 6) preventing relapse; and 7) preventing the development of PTSD in people whoare at high risk of non-recovery after a trauma [6]

Despite that theoretical principals, pharmacotherapy for PTSD has primarilybeen guided by empirical evidence that a specific drug has efficacy againsta specific symptom. Indeed, at present, very few data in treatment of psychiatric disorders,including PTSD, link psychobiological abnormalities to specific drug effects.In research and in clinical practice almost every class of psychotropicagent has been prescribed for PTSD patients. Most studies involve antidepressants:selective serotonin reuptake inhibitors (SSRIs), selective noradrenalin reuptake inhibitors (SNRIs), monoamine oxidaseinhibitors (MAOIs), tricyclic antidepressants (TCAs), and other serotonergicagents (trazodone and nefazodone). Antiadrenergic drugs tested include alpha-2 receptor agonists (clonidine and guanfacine) and the beta-receptor antagonist(propranolol).Other drugs studied include benzodiazepine, anxiolytics, and antipsychoticagents [7].

\section{Historical overview of the use of medicinal plants withadaptogenic and anti-stress effect}

The concept of the adaptogenic action was proposed by N.V. Lazarev in 1947 to describe the effects of arterial dilator dibazol-12-benzylbenzimidazole. Later this concept was developed through numerous experimental and clinical studies in the former USSR for substances that increase the body's resistance to various damaging factors of physical, chemical or biological nature. More than 1500 pharmacological and clinical studies were published in the USSR on the topic for adaptogenic activity by 1984. In 1998 the American Agency for Food and Drug Administration (FDA) and European Medicines Agency (EMA) adopted the concept of adaptogens as a term for evaluation of traditional herbal medicinal preparations (eg .: Ginseng spp., Eleutheroccocus spp., RhodiolaroseaL.,SchisandrachinensisL.andothers)[10].The use of adaptogens, however, dates back centuries in various traditional medical systems around the world. Ginseng is used in traditional Chinese medicine described as an aphrodisiac, stimulant and cure for diabetes. RhodiolaroseaL. or Golden root is traditionally used in the Siberian part of Russia and the Scandinavian countries to adapt to harsh climatic conditions. This plant has also been described in Chinese and Ayurveda medicine. Schisandrachinensis L. also is widely used in the northern parts of China and Russia as an adaptogen, which suppresses hunger, thirst,reduces fatigue and improves vision. 


\section{MECHANISMS OF ACTION OF PLANT PSYCHOTROPIC SUBSTANCES}

Mechanisms of action of plant medicines used to treat psychiatric disorders mainly include modulation of neuronal connections, through specific plant metabolites binding to neuromodulators receptors or through changes in the synthesis and the basic functions of neurotransmitters. Other mechanisms of action may include stimulating or inhibitory activity against CNS and regulating or supporting function to the endocrine system. Herbal medicines have a number of psychotherapeutic effects such as antidepressant, anxiolytic, nootropic (improving cognitive functions), and sedative, hypnotic and analgesic. Other traditionally observed effects that do not follow the standard terminology are adaptogenic and tonic action for which it is assumed that enhance adaptation to exogenous (external) stressors through a complex effect on the neurochemical and endocrine systems. Such effects can be clinically significant for a number of psychiatric disorders, including mood disorders, anxiety disorders and sleep disorders. Thus, the mechanisms underlying these disorders, although varied and reacted and often curing definition neurological, endocrine or chronobiologicalfactors beneficial effect can be observed in other areas. This could potentially affect the treatment of other co-morbid psychiatric disorders, e. if treating depression, anxiety may be dissipated or if treating insomnia depression can be relieved $[11,12]$.

One way to explore further details for the psychopharmacological effects of herbal medicines and to enhance their clinical validation is the use of "omic" genetic technologies. The "omic" technologies include pharmacogenomics, proteomics (epigenetics) and metabolomics. The use of the "omic" technologies in phytotherapy can be designated as herbomics. This technology can provide answers to pharmacodynamics, the toxicity / safety profile, the synergistic effects and clinical efficacy. One such use of "omic" technology is the study of epigenetic effects of herbal medicines by proteomic analysis. The results of two epigenetic studies conducted in the field of plant psychopharmacology reveal interesting data. Wong et al. (2004) conducted tests for gene expression in an animal model comparing 8 weeks administration of a single daily intravenous dose imipramine, Hypericumperforatum L. L., or saline as a control. The results showed that the herb differentially regulated 66 genes and the expression of their sequence, and imipramine regulate 74 . Six common transcript (affecting synaptic functions and features of the energetic metabolism) were expressed in both treatments [13]. Another proteomic analysis conducted on an animal model of Pennington et al. (2009) compared the gene expression of proteins with the use of Hypericumperforatum L. with the antidepressant clomipramine, as well as herbal combination of traditional Chinese medicine "CCIS - yao san" (XYS: used for mood disorders in Asia). From 1616 protein points analyzed Hypericumperforatum L. was found differentially expressed in 64 proteins in HT22 cells derived from rat hippocampal cell, XYS 40 protein and clomipramine 90. Mass spectrometry revealed that 43 protein points have been found overlapping expressions, most commonly affecting energy metabolism. Western-blot analysis showed that both herbal extracts and clomipramine unregulated expression of two forms of DRP - 2 protein involved in axonal growth and recovery, heat shock protein 70 (Hsp70) (neuronalprotein folding gene) also increases [14]. These studies provide evidence that Hypericumperforatum L. not only affects the transcription of many genes but modulates gene expression in the same manner as conventional antidepressant.Future similar proteomic studies of other plant psychotropic substances are promising to detect such genetically expressed similarities with conventional pharmacotherapies.

\section{HERBAL DRUGS WITH ANTIDEPRESSANT EFFECT}

Some herbal drugs with antidepressant action as Hypericumperforatum L. (St. John's wort), RhodiolaroseaL.(golden root), Crocus sativusL. (saffron) offer a promising treatment for PTSD by conventional psychopharmacological actions including inhibition of the secretion ofcatecholamines - serotonin, dopamine and norepinephrine, increased binding and sensitization of receptors to serotonin, inhibition of the enzyme monoamine oxidase (MAO) and influencing the neuro-endocrine modulation [15]. Suspected of involvement of GABA-ergic mediation and modulation of cytokine production (especially in major depressive disorder with concomitant inflammatory condition) and participation of opioid and cannabinoid systems. In most cases antidepressant mechanisms of action of herbal medicines are not as clearly defined as of SSRIs [16].

Some herbal drugs effects, improving mood (as $R$. rosea and $C$. sativus) also show anxiolytic effects. This may be due to modulation of the neural pathways mediating anti-depressant and anxiolytic effects (on GABA, serotonin and noradrenaline systems), or it may be due to the "halo effect" in which, effectively treating depression, anxiety, can also be reduced[17].

\section{HERBAL MEDICINES AFFECTING ANXIETY}

Anxiety disorders such as generalized anxiety disorder (GAD), social phobia and PTSD are characterized with an element of psychological anxiety and distress (American Psychiatric Association, 2000). The pathophysiology of anxiety disorders is still not clear, although the available data suggest that the neurobiology involves abnormalities in the serotonergic, noradrenaline, glutamate-ergic and GABA - ergic 
transmission [18]. The inclusion of these pathways is demonstrated by the efficacy of the inhibitors of serotonin reuptake inhibitors (SSRIs), selective serotonin and norepinephrine reuptake inhibitors (SNRIs) and benzodiazepines [19]. Phytotherapeuticals that may affect anxiety disorders as kava (Piper methysticumG.Forst.) is classified as "Anxiolytics", and generally have an effect on the GABA-ergic system [15], or operate by controlling the transmission in voltage-dependent ion channels, or by change in membrane conductivity, or by inhibiting GABA transaminase or glutamate decarboxylase or, less commonly by binding to benzodiazepine receptors (e.g. with $\alpha$-subunit) [20]. The subsequent stimulation of GABA-ergic neurotransmission has the effect of damping, which ultimately provides psychological calming effect [19].

In vitro analysis of brain homogenate of rats shows that the studied preparations aqueous extract of lemon balm (Melissa OfficinalisL.) manifests most strongly inhibiting GABA-transaminase activity, while chamomile (Matricariarecutita L.) and hops (HumuluslupulusL.) showed a significant inhibition of glutamate decarboxylase activity [21].

\section{Current phytotherapeutical options with adaptogenic and antidepressant action}

More than 60 years of clinical and biochemical studies of Rhodiolarosea L., elucidated the mechanisms by which plant extract exerts its therapeutic effects. Rhodiola rosea L. is a valuable medicinal herb known primarily as an adaptogen increasing the sustainability of the harmful effects of various stressors. Panossian et al, investigated the active substances from the extract of $R$. rosea (RR) and the possible synergistic effect of its active ingredients in animal models. Rats and mice that were repeatedly forced to swim in a confined space, such as behavioral characteristic result react with immobility. This endpoint reflects the state of despair was significantly affected by the substances that have proved that is therapeutically effective in human depression [22]. Mattioli et al. in one of their published findings that strongly showed that chronic administration of R.roseaL. results in potent inhibition of the behavioural and physiological changes induced by chronic exposure to mild stressors [23].R.roseaL. roots and rhizome contain 6 main classes of bioactive ingredients including phenylpropanoids, phenyletanoids derivatives, flavonoids, monoterpenes, triterpenes and phenolic acids. Rosavine, salidroside (rhodioloside) and tyrosol are used as markers to standardize the extracts from R.roseaL. [24]. In 1969 the pharmacological committee of the former Soviet Union recommends studiedRhodiolarosea L. in patients suffering from asthenic neuroses, hypotension, and schizophrenia and in healthy people dealing with intensive labor, requiring mental and physical endurance.Many systematic studies conclude that the standardized extract of Rhodiolarosea L. is useful in stress-induced fatigue. In a double-blind randomized placebo-controlled study ( 4 weeks) in patients with chronic fatigue syndrome, extract of RhodiolaroseaL. significantly reduced fatigue and improved concentration. Moreover, in healthy adults included in randomized clinical trials with exposure to stress, $R$. roseaL. demonstrated reduced fatigue andimproved memory functions [25].In another 6 week double-blind randomized placebo-controlled study of the use of $R$. rosea L. in patients with mild to moderate depression reported significant antidepressant effect compared to the control. They involved two groups, each of which is given the extract, respectively, in doses of $340 \mathrm{mg} /$ day and 680mg / day, and a third group receiving placebo. Both groups, of which the application treatment showed significant improvement in depression on the scale of Hamilton (Hamilton Depression Scale - HAMD), compared to placebo. The plant also reduces insomnia, emotional instability and improves self-esteem. In patients with different depression types treated in tricyclic antidepressants and extract of $R$. rosea $\mathrm{L}$. as adjunctive therapy reported reduced hospital stay. Addition of $R$. rosea L. in the therapy increases the activity and intellectual and physical endurance and reduces the side effects of tricyclicantidepressants [26].

\section{CONCLUSION}

In the scientific literature there is sufficient evidence in favor of that PTSD is characterized by specific psychobiological dysfunctions, which in turn are of interest in the use of differentdrug classes in the treatment of trauma-associated biological effects.Since PTSD is a chronic disorder, pharmacotherapy may continue for an indefinite period of time [8,9]. The use of novel phytotherapeuticals in the treatment and prevention of PTSD is supported from increasing number of studies. One future perspective for the pharmacotherapy in PTSD is finding the right combination of conventional antidepressant and adjuvant phytochemical substances.

\section{ACKNOWLEDGMENTS}

The authors declare that this work was supported by grant from the Medical University of Plovdiv - Project SDP15/2015.

\section{REFERENCES}

[1] Belmaker RH, Agam G. Major depressive disorder. N Engl J Med. Massachusetts Medical Society; 2008;358(1):55-68.

[2] Ressler KJ, Nemeroff CB. Role of serotonergic and noradrenergic systems in the pathophysiology of depression and anxiety disorders. Depression and Anxiety. 2000. p. 2-19. 
[3] Antonijevic IA. Depressive disorders - Is it time to endorse different pathophysiologies? Psychoneuroendocrinology. 2006. p. 1-15.

[4] Hindmarch I. Expanding the horizons of depression: Beyond the monoamine hypothesis. Human Psychopharmacology. 2001. p. 203-18.

[5] American Psychiatric Association. American Psychiatric Association, 2013. Diagnostic and statistical manual of mental disorders (5th ed.) [Internet]. American Journal of Psychiatry. 2013. 991 p.

[6] Steckler T, Risbrough V. Pharmacological treatment of PTSD - Established and new approaches. Neuropharmacology. 2012. p. 617-27.

[7] Zoellner LA, Feeny NC, Cochran B, Pruitt L. Treatment choice for PTSD. Behav Res Ther. 2003;41(8):879-86.

[8] Albucher RC, Liberzon I. Psychopharmacological treatment in PTSD: A critical review. Journal of Psychiatric Research. 2002. p. 355-67.

[9] Cukor J, Spitalnick J, Difede J, Rizzo A, Rothbaum BO. Emerging treatments for PTSD. Clinical Psychology Review. 2009. p. 715-26.

[10] Doc. Ref. EMEA/HMPC/102655/2007. Emea. European Medicines Agency. 2014;(May). Available from: http://www.ema.europa.eu/ema/

[11] Panossian AG. Adaptogens in Mental and Behavioral Disorders. Psychiatric Clinics of North America. 2013. p. 49-64.

[12] Sarris J, Panossian A, Schweitzer I, Stough C, Scholey A. Herbal medicine for depression, anxiety and insomnia: A review of psychopharmacology and clinical evidence. European Neuropsychopharmacology. 2011. p. 841-60.

[13] Wong M-L, O'Kirwan F, Hannestad JP, Irizarry KJL, Elashoff D, Licinio J. St John's wort and imipramine-induced gene expression profiles identify cellular functions relevant to antidepressant action and novel pharmacogenetic candidates for the phenotype of antidepressant treatment response. Mol Psychiatry [Internet]. 2004;9(3):237-51

[14] Pennington K, Föcking M, McManus C a, Pariante CM, Dunn MJ, Cotter DR. A proteomic investigation of similarities between conventional and herbal antidepressant treatments. J Psychopharmacol [Internet]. 2009;23(5):520-30

[15] Sarris J. Herbal medicines in the treatment of psychiatric disorders: a systematic review. Phytother Res. 2007;21:703-16.

[16] Coutts RT. The Psychopharmacology of Herbal Medicine: Plant Drugs That Alter Mind, Brain and Behavior [Internet]. Journal of Psychiatry and Neuroscience. Canadian Medical Association; 2003 [cited 2016 May 31]. p. 300.

[17] Brady KT, Verduin ML. Pharmacotherapy of comorbid mood, anxiety, and substance use disorders. Subst Use Misuse. 2005;40(13-14):2021-41, 2043-8.

[18] Nutt DJ, Ballenger JC, Sheehan D, Wittchen H-U. Generalized anxiety disorder: comorbidity, comparative biology and treatment. Int J Neuropsychopharmacol. 2002;5:315-25.

[19] Baldwin DS, Waldman S, Allgulander C. Evidence-based pharmacological treatment of generalized anxiety disorder. Int J Neuropsychopharmacol [Internet]. 2011;14(05):697-710.

[20] Sarris J, Kavanagh DJ, Byrne G, Bone KM, Adams J, Deed G. The Kava Anxiety Depression Spectrum Study (KADSS): A randomized, placebo-controlled crossover trial using an aqueous extract of Piper methysticum. Psychopharmacology (Berl). 2009;205(3):399-407.

[21] Awad R, Muhammad A, Durst T, Trudeau VL, Arnason JT. Bioassay-guided fractionation of lemon balm (Melissa officinalis L.) using an in vitro measure of GABA transaminase activity. Phyther Res. 2009;23(8):1075-81.

[22] Panossian A, Nikoyan N, Ohanyan N, Hovhannisyan A, Abrahamyan H, Gabrielyan E, et al. Comparative study of Rhodiola preparations on behavioral despair of rats. Phytomedicine. 2008;15(12):84-91.

[23] Mattioli L, Funari C, Perfumi M. Effects of Rhodiola rosea L. extract on behavioural and physiological alterations induced by chronic mild stress in female rats. J Psychopharmacol [Internet]. 2009;23(2):13042. Available from: http://www.ncbi.nlm.nih.gov/pubmed/18515456

[24] Panossian A, Wikman G, Sarris J. Rosenroot (Rhodiola rosea): Traditional use, chemical composition, pharmacology and clinical efficacy. Phytomedicine. 2010. p. 481-93.

[25] Shevtsov V a, Zholus BI, Shervarly VI, Vol'skij VB, Korovin YP, Khristich MP, et al. A randomized trial of two different doses of a SHR-5 Rhodiola rosea extract versus placebo and control of capacity for mental work. Phytomedicine. 2003;10:95-105.

[26] Olsson EMG, Von Schéele B, Panossian AG. A randomised, double-blind, placebo-controlled, parallelgroup study of the standardised extract SHR-5 of the roots of Rhodiola rosea in the treatment of subjects with stress-related fatigue. Planta Med. 2009;75(2):105-12. 